\title{
Metrology with Simple Atoms: Fundamental Constants of Physics
}

\section{Massimo Inguscio and Marco Prevedelli University of Florence, Italy}

European laboratories are leading in research where methods of laser spectroscopy are applied to the study of simple atoms. Ultra precise measurements and a comparison with accurate theoretical calculations allow the determination of fundamental quantities such as the Rydberg constant Ry, the fine structure constant $a$ and the r.m.s. charge radius of the proton rp. Stringent tests of QED for bound systems have also been performed

Over the last few years rapid advances in non-linear optics, optical frequency metrology and laser stabilisation techniques have stimulated ultra-high resolution spectroscopic measurements at wavelengths not accessible in the past. Direct

Fig 1 Recording of the two photon transitions on rubidium on which the new optical frequency standard developed in Paris is based

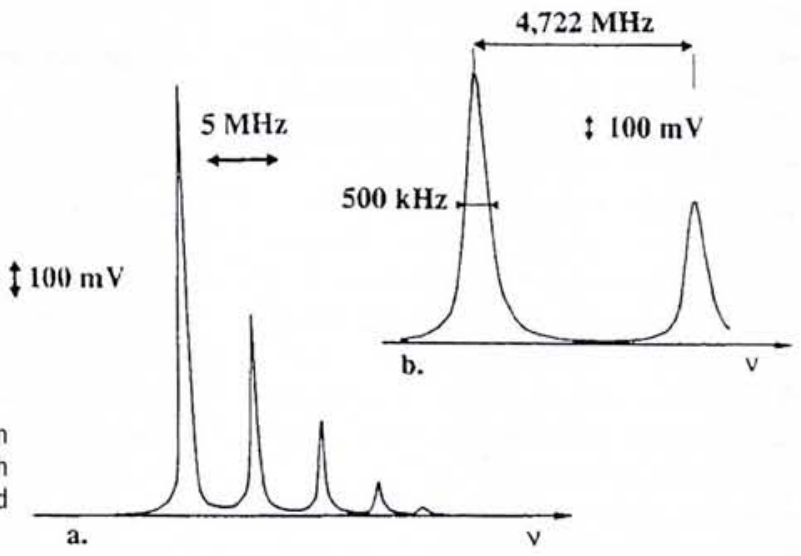

comparison of optical frequencies with the caesium frequency standard can lead to a resolution $\Delta v / v$ of the order of $10^{-13}$. However, these experiments pose formidable technical challenges, and it is only worthwhile to work on them if the frequency for the transitions to be measured can be computed with comparable accuracy from first principles. In that case, most of the amazing experimental accuracy can then be transferred to fundamental constants. Alternatively, the experiment can be considered as a stringent test of theory. The requirement for high theoretical accuracy basically excludes all but the simplest atoms, ie hydrogen and helium.

\section{Hydrogen Experiments}

Recent accurate two-photon spectroscopy on the hydrogen atom reduced the relative uncertainty in the determination of the Rydberg constant $\mathrm{R}_{\mathrm{y}}$, which determines the natural energy scale of atomic and molecular physics, by a factor of 300 over the last two decades. This progress is almost entirely due to efforts by the groups of the Ecole Normal Supérieure in Paris (F. Biraben, B. Cagnac and L. Julien) and at the Max Planck Institute for Quantum Optics in Garching (T.W. Hänsch, previously at Stanford).

In Paris the frequencies of $2 \mathrm{~S}-n \mathrm{~S}$ or $2 \mathrm{~S}$ $n \mathrm{D}$ two-photon transitions with $n$ around 10 , are studied. These transitions conveniently fall within the range of continuous wave Ti:Sapphire lasers. The main advantage of these transitions is that the uncertainty introduced by QED and finite atoms, to name just a few of these developments.

One of the most striking consequences of the wave-like behaviour of atomic matter is the observation of interference effects with atoms. Similar to light in regular optical interferometry an atomic wave packet can take two separate paths from the input port of an atom interferometer to its output port. Depending on the phase difference experienced between the two legs constructive or destructive interference can be observed. A continuous change in the phase difference causes a periodic change between both cases, leading to interference fringes. Besides important studies concerning the very nature of quantum mechanics, atomic physics and quantum optics, atom interferometers are already in use as highly sensitive measurement instruments. In addition to the realization of a new generation of atomic clocks, atom interferometers can be used to study a broad range of external potentials acting on atoms, one example being an interferometer capable of measuring variations in Earth's gravity with a resolution of $\Delta \mathrm{g} / \mathrm{g}$ of the order of $10^{-9}$.

Atomic physics is clearly one of the branches of physics that profited the most from the invention of the laser. The field of controlled manipulation of atoms wouldn't have evolved anywhere close to its present stage if laser light had not been at hand. As described above, laser manipulation is one of the key ingredients in revealing the wave-like behaviour of atomic matter, also manifesting itself so impressively in the observation of Bose-Einstein condensation. A condensate has already been used as a reservoir for a pulsed laser-like source of atoms. The same leap forward that was caused by switching from ordinary light sources to laser light is expected to occur with the development of a continuouswave laser-like source of atoms. Laser manipulation of atoms will have the opportunity of paying back all the benefits it received from regular lasers by developing laser-like atoms sources which may find similarly fruitful applications in other branches of physics.
Further Reading

W.D. Phillips Experiments in Laser Cooling "Fundamental Systems in Quantum Optics" Les Houches Summer School 1990, J. Dalibard, J.-M. Raimond, and J. Zinn-Justin (eds.); Elsevier 1992

C. Cohen-Tannoudji Theory of Laser Cooling "Fundamental Systems in Quantum Optics" Les Houches Summer School 1990, J. Dalibard, J.-M. Raimond, and J. Zinn-Justin (eds.), Elsevier 1992

K. Sengstock and W. Ertmer "Advances in Atomic, Molecular and Optical Physics" vol 35, Academic Press 1995

C.S. Adams, M. Sigel, J. Mlynek Atom Optics and Atom Interferometry Physics Reports vol 240, 1994 
nuclear size effects are small. As these effects scale as $n^{-3}$ only a modest theoretical accuracy is required for the upper levels, whereas a precise experimental value is available for the $2 \mathrm{~S}$ Lamb shift.

Technical progress towards a pure frequency measurement involved the development of a now widely applied secondary frequency standard in the visible, using the $5 \mathrm{~S}_{1 / 2}-5 \mathrm{D}_{5 / 2}$ two-photon transition in $\mathrm{Rb}$ at $778 \mathrm{~nm}$ (figure 1). In the most recent experiment the $2 \mathrm{~S}-8 \mathrm{D}$ transition at $770 \mathrm{THz}$ in hydrogen and deuterium were investigated, resulting in a $1 \sigma$ uncertainty of less than $5 \mathrm{kHz}$. The final, impressive value for the Rydberg constant is:

$R_{y}=10973731.56859$ (10) $\mathrm{m}^{-1}$ The uncertainties due to frequency measurement and Lamb shift calculations are about equal $\left(6.4 \times 10^{-12}\right.$ and $7.7 \times 10^{-12}$ respectively) so further progress needs both theoretical and experimental improvements.

At Garching the two-photon $1 \mathrm{~S}-2 \mathrm{~S}$ transition has been studied. This research was started by T.W. Hänsch more

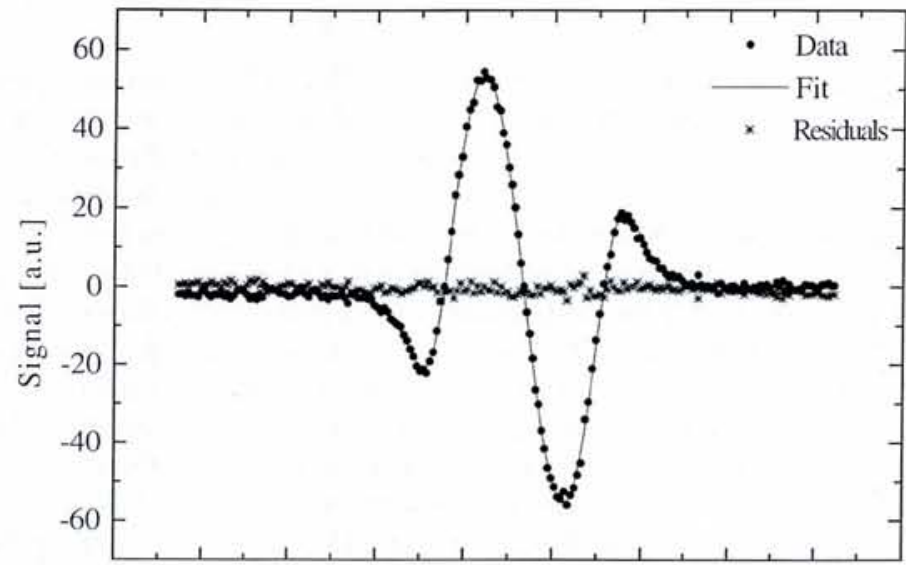

31800318103182031830318403185031860318703188031890 further 10 per cent, and a highly precise value for the $1 S$ Lamb shift can be deduced:

$$
\mathrm{L}_{1}=8172.876(29) \mathrm{MHz}
$$

Alternatively, assuming QED calculations to be correct, the r.m.s. radius of an elementary particle, the proton, can be determined:

$$
r_{p}=0.890(14) \mathrm{fm}
$$

This last result shows how a small-sized experiment in atomic physics can measure properties of elementary particles. cy of $15 \mathrm{kHz}$ (possibly $1 \mathrm{kHz}$ can be reached) in the calculation of the fine structure intervals in the $2{ }^{3} \mathrm{P}$ level. A precision measurement of the fine structure splitting (about $30 \mathrm{Ghz}$ ) and comparison with theory then gives a value of $\alpha$ with an accuracy comparable to results form other branches of physics. In Florence we observe the $2{ }^{3} \mathrm{~S}-2{ }^{3} \mathrm{P}$ transition at $1083 \mathrm{~nm}$, now conveniently accessible with diode lasers. The precision in the measurement of fine structure intervals so far is about $2.5 \mathrm{kHz}$. A typical example of an experimental signal is shown in figure 3. Further work is in progress to analyse systematic effects.

In conclusion, high resolution spectroscopy of simple atoms can provide highly precise values for fundamental constants. These results are of broad interest even outside the spectroscopic community. In particular it has been demonstrated that quantities usually considered to be part of the realm of particle physics, such as rp, can be accurately determined with

than twenty years ago; recently his group succeeded in the first pure frequency measurement of this ground state transition both for hydrogen and deuterium. This transition is most sensitive to effects not included in the Dirac theory. The $1 \mathrm{~S}$ Lamb shift is about 1 Ghz, whereas the shift induced by the finite size of the proton is $\sim 0.5 \mathrm{Mhz}$. The study of the $1 \mathrm{~S}-2 \mathrm{~S}$ transition, which is complementary to the study performed by the Paris group, required the development of a powerful source of highly monochromatic ultraviolet radiation at $243 \mathrm{~nm}$. Thanks to the growth of high-quality, non-linear crystals such a source recently became possible. Using the coldest atoms of a beam of hydrogen atoms at a temperature of $6 \mathrm{~K}$ the transition was recorded with a FWHM of less than $1 \mathrm{Khz}$ at a frequency of 2466 Thz. Although the natural linewidth is still three orders of magnitude smaller, this result determines a new record in high resolution optical spectroscopy. The experimental value for the transition frequency, eliminating hyperfine structure contributions, is:

$\nu_{1 \mathrm{~s}}-\nu_{2 \mathrm{~s}}=2446061413187.34(84) \mathrm{kHz}$ with a relative uncertainty as low as $3.4 \times 10^{-14}$. Combining the Garching and Paris results improves the value of $R_{y}$ by a
Fig 2Third derivative measurement and fit for the $\mathrm{J}=2$, $\mathrm{J}=0$ fine structure interval of the $2^{3} \mathrm{P}$ level in helium

\section{Helium Experiments}

The helium atom can now compete with the hydrogen atom for the measurement of fundamental constants. This is due both to improvements in experiment and theory. For many years laser spectroscopy of He was limited to excitations from metastable atoms only because the $1{ }^{1} \mathrm{~S}-2$ ${ }^{1} \mathrm{P}$ transition at $58 \mathrm{~nm}$ was out of reach for laser sources. Recently, the group of W. Hogervorst at the Vrije Universiteit in Amsterdam succeeded in generating the $10^{\text {th }}$ harmonics of a narrowband $584 \mathrm{~nm}$ dye laser. They recorded the ground state transition with a linewidth of $600 \mathrm{Mhz}$ (natural width $300 \mathrm{Mhz}$ ) and measured the absolute frequency for the $1{ }^{1} \mathrm{~S}-2{ }^{1} \mathrm{P}$ transition with an accuracy of $45 \mathrm{Mhz}$. This corresponds to a $\Delta \nu / \nu$ of $9 \times 10^{-9}$. The theoretical frequency, which includes a 1 ${ }^{1} S$ Lamb shift-contribution of about $41 \mathrm{Ghz}$ and is calculated with an accuracy of $35 \mathrm{Mhz}$, is in good agreement with experiment.

The helium atom also can be used to accurately determine the fine structure constant $\alpha$. Theory now claims an accura- tabletop-sized experiments. These experiments, although highly specialised, have produced technological breakthroughs which are of general use in non-linear optics and optical metrology.

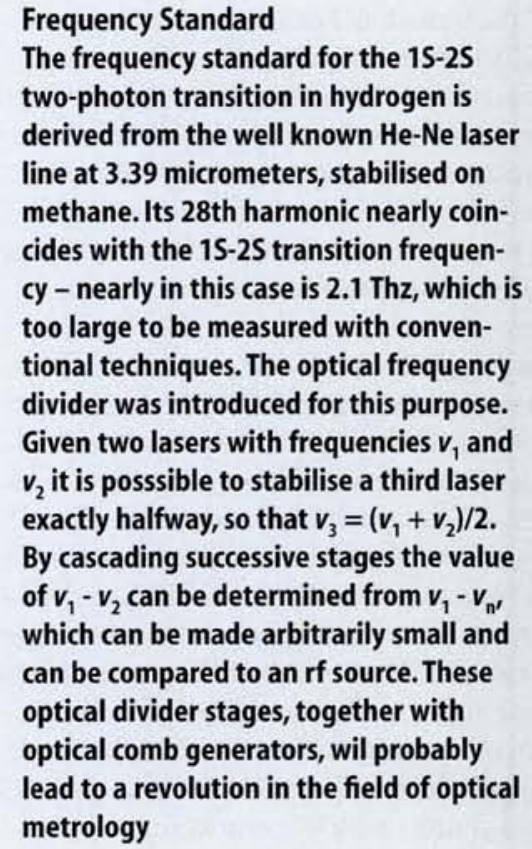

